\title{
The Transition State in Chemical Kinetics
}

\section{By Prof. M. Polanyi, University of Manchester}

$\mathrm{T}^{\mathrm{H}}$ HE energy barrier separating the initial and final states of a reaction has ceased to be a fictitious concept since the main principles of chemical inertia were discovered by $\mathrm{F}$. London (1927). For some simple cases, we can now calculate the energy changes occurring during the process of reaction, and follow the paths along which the atoms move. We can, in particular, obtain in many cases a clear picture of the configuration which corresponds to the top of the energy barrier. This configuration is called the transition state $\tau$ of the reaction.

It is interesting to deduce Arrhenius's equation by thermodynamical considerations relating to the transition state. At chemical equilibrium we see the atomic configuration changing forwards and backwards between the initial and final states of a reaction, and passing on each occasion through the transition state $\tau$. The positions which the atomic system takes up between the initial and final states form a continuous sequence and can, therefore, be defined by one co-ordinate $l$ which is called the 'reaction path'. At equilibrium we have a certain infinitesimal number of reacting systems in the transition state, that is, near the top of the energy barrier. This number (population) can be equated to the concentration product of the reactants, say, $c^{\prime} . c^{\prime \prime}$... into an infinitesimal equilibrium constant $K_{1} d l$.

Since

$$
\text { Population }(\tau)=c^{\prime} \cdot c^{\prime \prime} \ldots K_{1} d l
$$

Population $=$ birth-rate $\times$ average life, we can calculate the birth-rate by noting that the average life of $\tau$ is equal to a thermal velocity $v$ over $d l$, and that thus:

$$
\text { Birth-rate }(\tau)=c^{\prime} . c^{\prime \prime} \ldots K_{1} v \text {. }
$$

Now the birth-rate of $\tau$ is equal to the rate of the forward reaction (1) plus the rate of the back reaction (2), and since at equilibrium, rate $(1)=$ rate (2)

$$
\text { rate }(1)=c^{\prime} \cdot c^{\prime \prime} \ldots K_{1} v / 2
$$

and the rate constant $k_{1}$ is

$$
k_{1}=K_{1} v / 2 \ldots(\mathrm{A})
$$

Applying van t'Hoff's isochor to $K_{1}$, we obtain :

$$
\frac{d \log k_{1}}{d T}=-\frac{q}{R T}+\frac{d \log v / 2}{d T} .
$$

The second term on the right being negligible, this gives $k_{1} \propto e^{-q_{1} / R T}$, that is, Arrhenius's equation.
The 'transition state method' of treating problems of reaction velocity consists in a general. ization of this procedure to other variations than those of temperature. Supposing the reaction rate in solution changes under the influence of hydro. static pressure $\pi$, then applying thermodynamics to equation (A) we obtain

$$
\frac{d \log k_{1}}{d \pi}=\frac{V_{1}-V_{\tau}}{R T}+\frac{d \log v / 2}{d \pi},
$$

where $V_{1}$ is the volume of a solution containing one mol of the initial state and $V_{1}$ the volume of a solution containing one mol of the transition state. We can again neglect the differential of $v$ and set

$$
k_{1} \propto e^{\left(\nabla_{1}-\nabla r\right) / R T}
$$

The fruitfulness of this deduction depends on our capacity to interpret the values of $V_{1}-V_{\tau}$ derived from observation. This is easiest in the case of reactions of the type $A+B=A B$; for these we can predict $V_{1}-V_{\tau}$ with some accuracy. The configuration of the transition state must here be intermediate between the configuration of the initial and final states and quite near to that of the final state, and the same will hold for the volume $V_{\tau}$. $V_{\tau}$ will lie between $V_{1}$ and $V_{2}$ (the volume of the final state) close to $V_{\mathrm{z}}$. This has, in fact, been confirmed by experiment. It thus seems that the rate at which reaction velocity varies with hydrostatic pressure is predictable for certain types of reactions.

The method is capable of further generalization. Variations in reaction velocity can be observed under the influence of an electric field, for example, when the rate of electrolytic evolution of hydrogen is governed by the polarization $(\varepsilon)$ of an electrode. In this case we might write for the equilibrium constant $K$ which relates the concentration of the hydrogen ions in solution to that of the hydrogen gas at the electrode,

$$
\frac{d \log K}{d \varepsilon}=\frac{n}{R T},
$$

where $n$ is the effective (molar) charge of the proton. The effective charge is equal to $F=96,500$ coulombs for the hydrogen ion which constitutes the initial state, and equal to zero for the final state when the proton is neutralized at the electrode. If we assume that in the transition state 
the effective charge has an intermediate value, say, $\alpha n, \alpha$ being about $0 \cdot 5$, we obtain

$$
\frac{d \log }{d \varepsilon} \frac{K_{1}}{R T}=\frac{\alpha F}{R T} ; \alpha \sim 0.5 ;
$$

which on comparison of the differential of equation A with respect to $\varepsilon$ and by neglecting $\frac{d \log v / 2}{d \varepsilon}$ can be identified with $\frac{d \log k_{1}}{d \varepsilon}$. If we measure $k_{1}$ by the intensity $i$ of the current, we have $\log k_{1}$ $\propto \log i$ and finally

$$
\log i=\frac{\alpha F_{\varepsilon}}{R T}+\text { const. ; } \alpha \sim 0.5 ;
$$

which is the well-known over-voltage equation of Tafel. This equation is thus interpreted as indicating that the effective charge of the transition state lies about half-way between that of the initial and the final states of the reaction.

The field of such generalizations has been extended to other variations of reaction velocity arising from changes in chemical constitution, from the transfer of a reaction from the gas phase into solution, and from variations of solvents.
The principle consists in introducing a parameter of the transition state arising from the general equation

$$
\frac{d \log k_{1}}{d \chi}=\frac{p_{1}-p_{\tau}}{R T}
$$

where $\chi$ is an external parameter causing the variation in reaction rate and $p_{1}-p_{\tau}$ is the change in the corresponding parameter accompanying the formation of the transition state from the initial state.

The advance in the various directions to which I have referred is, as yet, tentative, but the method seems to provide a rational framework for the interpretation of many empirical rules of reactivity as well as a guidance to the discovery of new relationships.

\section{REFERENCES}

The derivation of a reaction velocity from a statistical treatment of the transition state was first put forward by Wigner and Pelzer (Z. phys. Chem., B, 15, 445; 1932) in their treatment of the reaction $\mathrm{H}+\mathrm{H}_{2}$ para $=\mathrm{H}_{2}+\mathrm{H}$; this treatment was generalized by Eyring (J. Chem. Phys., 3, 107; 1935) and by Evans and Polanyi (Trans. Far. Soc., 31, 875; 1935). The thermodynamic formulation equation $(A)$ is due to the latter authors and to Wynne-Jones and Eyring $(J$. Chem. Phys., 3, 492; 1935). Equation $(B)$ and its various applications sketched out in this paper are due to Evans and Polanyi (Trans Far. Soc., 31, 875; 1935; 32, 1333; 1936).

\section{Obituary Notices}

\section{Sir Albert Kitson, C.M:G., C.B.E.}

GIR ALBERT KITSON, whose death occurred on $\checkmark$ March 8, was a geologist of world-wide repute, and the discoveries which he made and which are now being exploited in many parts of the world entitle him to be classed as one of the foremost economic geologists of his time.

Kitson was born in Manchester in 1868, but when six years old accompanied his parents to Australia. $\mathrm{He}$ entered the Civil Service of Victoria by competitive examination, and having a bent for geology he took courses in this subject at the University and School of Mines, Melbourne. Later on, he also took courses in mining and surveying, thereby equipping himself for the career which he was afterwards to follow.

As a result of his studies, Kitson was transferred to the Geological Survey of Victoria. In 1903 he became senior geologist, and while he occupied this post was responsible for some magnificent field work. In 1906 he went to Southern Nigeria as principal of the Mineral Survey, but resigned in 1911. In 1915 he was appointed director of the Geological Survey of the Gold Coast, which position he held until 1930 when he retired, having reached the age limit. So valuable were his services considered, however, that his period of service was extended five years beyond the usual period. It was while he occupied this position that his name became known in ever- widening circles, not only on account of the remarkable discoveries which have already been referred to, but also on account of the energy and drive which he put into his work. By many of the Gold Coast natives he was regarded as a fetish doctor owing to his seemingly reckless handling of snakes, an ability which he had acquired during his boyhood in the Australian bush. Nor did he know what fear meant. In the early days of his Southern Nigerian appointment, when he was geologizing in the wake of a punitive expedition, he was continually in trouble with the military authorities as he was always getting ahead of them.

Among Kitson's discoveries on the west coast of Africa may be mentioned the black and brown coal. fields (Nigeria), manganese, bauxite, diamonds (Gold Coast) and many others of lesser importance, the development of which has contributed enormously to the prosperity of these two colonies. But he followed up these discoveries by coming home and describing their possibilities to interested parties in the business world of London for, as already indicated, he had the economic side of geology very strongly developed. Moreover, he was a great enthusiast and he managed by some subtle gift to impart his enthusiasm to others.

After his retirement, Kitson was requested by the Government of Kenya to carry out a preliminary geological survey with special reference to the 